\title{
NMDA Receptor Blockade by Ketamine Abrogates Lipopolysaccharide-Induced Depressive-Like Behavior in C57BL/6J Mice
}

\author{
Adam K Walker 1,4, David P Budac², Stephanie Bisulco ${ }^{3}$, Anna W Lee ${ }^{3}$, Robin A Smith', Brent Beenders', \\ Keith W Kelley' and Robert Dantzer ${ }^{*, 1,4}$ \\ 'Integrative Immunology and Behavior Program, Department of Animal Sciences and Department of Medical Pathology, University of Illinois at \\ Urbana-Champaign, Urbana, IL, USA; '2Department of Bioanalysis and Physiology, Lundbeck Research USA, Paramus, NJ, USA; \\ ${ }^{3}$ Neuroinflammation Disease Biology Unit, Lundbeck Research USA, Paramus, NJ, USA; ${ }^{4}$ Division of Internal Medicine, Department of Symptom \\ Research, The University of Texas MD Anderson Cancer Center, Houston, TX, USA
}

We have previously demonstrated that lipopolysaccharide (LPS) induces depressive-like behavior by activating indoleamine 2,3 dioxygenase (IDO; O'Connor et al, 2009c). IDO degrades tryptophan along the kynurenine pathway. Using mass-spectrometry (LC-MS) analysis of kynurenine metabolites in the brain of mice injected at the periphery with I mg/kg LPS, we show that LPS activates the kynurenine 3-monooxygenase pathway that ultimately degrades kynurenine into quinolinic acid. As quinolinic acid acts as an $\mathrm{N}$-methyl-D-aspartate (NMDA) receptor agonist, we used the NMDA receptor antagonist ketamine to assess the role of NMDA receptor activation in LPS-induced depressive-like behavior. Here, we report that a low dose of ketamine $(6 \mathrm{mg} / \mathrm{kg}$, intraperitoneally) immediately before administration of LPS $(0.83 \mathrm{mg} / \mathrm{kg}$, intraperitoneally) in C57BI/6 J mice abrogated the development of LPS-induced depressive-like behavior, without altering LPS-induced sickness measured by body weight loss, decreased motor activity, and reduced food intake. Depressive-like behavior was measured $24 \mathrm{~h}$ after LPS by decreased sucrose preference and increased immobility in the forced swim test (FST). Ketamine had no effect on LPS-induced cytokine expression in the liver and brain, IDO activation, and brainderived neurotrophic factor (BDNF) transcripts. The ability of ketamine to abrogate LPS-induced depressive-like behavior independently of a possible interference with LPS-induced inflammatory signaling was confirmed when ketamine was administered I0 h after LPS instead of immediately before LPS. In contrast, ketamine had no effect when administered $24 \mathrm{~h}$ before LPS. To confirm that NMDA receptor antagonism by ketamine mediates the antidepressant-like activity of this compound in LPS-treated mice, mice were pretreated with the $\alpha$-amino-3-hydroxy-5-methylisoxazole-4-propionic acid (AMPA) receptor antagonist 2,3-dihydroxy-6-nitro-7-sulfamoylbenzo(f)quinoxaline-2,3-dione (NBQX) to block enhanced AMPA receptor glutamatergic neurotransmission after NMDA receptor antagonism by ketamine. NBQX administered at the dose of $10 \mathrm{mg} / \mathrm{kg}$ intraperitoneally 15 min before ketamine in mice treated with LPS $24 \mathrm{~h}$ earlier restored LPS-induced decreased sucrose preference. These findings indicate that LPS-induced depressive-like behavior is mediated by NMDA receptor activation, probably as a consequence of formation of quinolinic acid.

Neuropsychopharmacology (2013) 38, 1609-1616; doi:10.1038/npp.2013.71; published online 3 April 2013

Keywords: ketamine; depression; NMDA receptor; AMPA receptor; lipopolysaccharide; inflammation

\section{INTRODUCTION}

Inflammation induces depressive symptoms in at-risk individuals (Capuron et al, 2002; Reichenberg et al, 2001; Wright et al, 2005). Analysis of the time course of symptoms of depression in patients treated chronically with interferon- $\alpha$ demonstrates that the onset of psychological and cognitive symptoms (for example, depressed

*Correspondence: Dr Robert Dantzer, Division of Internal Medicine, Department of Symptom Research, The University of Texas MD Anderson Cancer Center, I 400 Pressler Road Unit I 450, Houston, TX 77030, USA, Tel: +I 713563 4793, Fax: + 713745 3475, E-mail: rdantzer@mdanderson.org

Received 22 March 2012; revised 8 March 2013; accepted 8 March 2013; accepted article preview online 19 March 2013 mood, anxiety, and cognitive dysfunction) occurs much later than the onset of neurovegetative symptoms (for example, loss of appetite, anorexia, pain, and fatigue; Capuron et al, 2002). In addition, pretreatment by the specific serotonin reuptake inhibitor, paroxetine, abrogates the development of the psychological and cognitive symptoms but has little or no effect on neurovegetative symptoms (Capuron et al, 2002). The neurovegetative symptoms of depression are similar to the behavioral signs of sickness that can be observed in rodents of which the peripheral immune system has been activated (Dantzer et al, 2008). Using preclinical models of inflammationinduced behavioral alterations, we have confirmed that depressive-like behavior develops over a background of sickness and its mechanisms can be dissociated from 
sickness (Frenois et al, 2007; Moreau et al, 2008). The switch from sickness to depression is mediated by activation of the tryptophan-degrading enzyme indoleamine 2,3 dioxygenase (IDO). More specifically, genetic or pharmaceutical blockade of IDO abrogates the onset of depressive-like behavior without affecting sickness (O'Connor et al, 2009b). IDO activation results in an increase in the kynurenine/tryptophan ratio that occurs both at the periphery and in the brain. Brain kynurenine is degraded further into kynurenine metabolites, the nature of which depends on the brain cell types that are activated. Systemic inflammation induced by intraperitoneal injection of the cytokine inducer lipopolysaccharide (LPS) activates brain perivascular macrophages and microglia (Serrats et al, 2010; van Dam et al, 1992). Microglia express kynurenine 3-monooxygenase that degrades kynurenine into quinolinic acid, whereas astrocytes express kynurenine aminotransferases that lead to the formation of kynurenic acid (Guillemin et al, 2001; Heyes et al, 1996). Quinolinic acid acts as an $N$-methyl-D-aspartate (NMDA) receptor agonist, whereas kynurenic acid has NMDA antagonism properties. In response to systemic immune activation induced by intraperitoneal administration of pokeweed mitogen, the brain formation of quinolinic acid predominates over that of kynurenic acid (Saito et al, 1992). On the basis of these previous findings, we hypothesized that the IDO-dependent development of depressive-like behavior in response to LPS is mediated by the increased formation of brain quinolinic acid and the subsequent activation of NMDA receptors. In accordance with this hypothesis, we demonstrate in the present report that LPS activates the metabolic pathway, which leads to the formation of quinolinic acid in the brain, and that ketamine, a specific antagonist of the NMDA receptor, blocks LPSinduced depressive-like behavior by redirecting increased glutamatergic neurotransmission toward $\alpha$-amino-3-hydroxy-5-methyl-4-isoxazolepropionic acid (AMPA) receptor activation.

\section{MATERIALS AND METHODS}

\section{Animals and Treatments}

CD-1 (6 weeks old, $n=20$; Charles River) and C57BL/6J male mice ( 12 weeks old, $n=164$; The Jackson Laboratory) were used in these experiments. Mice were housed individually in standard shoebox cages in a temperature $\left(23{ }^{\circ} \mathrm{C}\right)$ and humidity (45-55\%)-controlled environment, with a 12/12-h modified dark-light cycle (lights on at 2200 hours). Food and water were available ad libitum. Mice were handled daily for 1 week before experimentation.

On the day of injection, fresh solutions of LPS (L-020M4062, serotype 0127:B8; Sigma, St Louis, MO) and ketamine (Fort Dodge Animal Health, USA) were prepared with sterile endotoxin-free isotonic phosphate-buffered saline (PBS), and administered intraperitoneally. Mice received either $6 \mathrm{mg} / \mathrm{kg}$ ketamine or sterile endotoxin-free isotonic saline (equivolume). This dose of ketamine was chosen on the basis of its ability to block LPS-induced depressive-like behaviors with minimal off-target effects, as determined from a dose-response pilot study. With the exception of experiment 1, where $1 \mathrm{mg} / \mathrm{kg}$ of LPS was injected intraperitoneally, mice were injected with either
$0.83 \mathrm{mg} / \mathrm{kg}$ of LPS or sterile endotoxin-free isotonic saline (equivolume) via the same route, immediately following ketamine or saline injections. This dose of LPS was chosen for its ability to reliably induce the acute sickness response and subsequent depressive-like behaviors across the time points examined here (Mormède et al, 2004; O'Connor et al, 2009c).

At the end of the experiment, mice were euthanized after $\mathrm{CO}_{2}$ anesthesia. Blood was collected by cardiac puncture and brains were removed after perfusion with sterile PBS. All biochemical assays on brain tissue were conducted on entire half brains cut down the midline to include a representation of all brain regions. All protocols in this study were approved either by the Institutional Animal Care and Use Committees of the University of Illinois, Texas A\&M University or Lundbeck Research USA.

\section{Experiment 1: LPS-Induced Production of Kynurenine Metabolites}

Experiment 1 was conducted in collaboration at Lundbeck Research USA (Paramus, New Jersey). To determine the exact nature of LPS on kynurenine metabolites, CD-1 mice were injected with $1 \mathrm{mg} / \mathrm{kg}$ LPS (L-3129, serotype 0127:B8; Sigma) or with sterile endotoxin-free isotonic PBS (equivolume) intraperitoneally. Brains were collected $24 \mathrm{~h}$ thereafter, following $\mathrm{CO}_{2}$ anesthesia and liquid chromatography coupled with LC-MS (see Supplementary Material) was used to determine brain levels of tryptophan, kynurenine metabolites, serotonin, and 5-hydroxyindoleacetic acid (5-HA).

\section{Experiment 2: Effects of Ketamine on LPS-Induced Behavioral and Biochemical Alterations}

As the antidepressant properties of ketamine can be confounded by the ability of this compound at high dosages to upregulate heme-oxygenase 1 , an inducible enzyme that has potent anti-inflammatory and antioxidant properties (Hoetzel and Schmidt, 2010), we first selected a dose of ketamine that is devoid of such activity. In view of the ability of ketamine to increase brain-derived neurotrophic factor (BDNF) at antidepressant doses (Autry et al, 2011), we also checked that ketamine does not interfere with the decrease in BDNF that has been reported in response to LPS (Guan and Fang, 2006; Wu et al, 2011).

All behavioral experiments were performed during the first $5 \mathrm{~h}$ of the dark phase of the light cycle. Figure 1 outlines the experimental design and timing of behavioral experiments. Specific details of behavioral and biochemistry assays can be found in Supplementary Material.

Locomotor activity. LPS-induced changes in locomotor activity were assessed 2 and $24 \mathrm{~h}$ after drug administration for mice allocated to depressive-like behavioral assessment (experiment 2A). Mice allocated to tissue collection $6 \mathrm{~h}$ after drug administration were assessed for LPS-induced changes in activity $5.5 \mathrm{~h}$ after treatment (experiment $2 \mathrm{~B}$ ).

Sucrose preference. Mice were trained for optimal sucrose preference (1\% solution) before testing. Sucrose preference testing was conducted immediately after locomotor assessment $24 \mathrm{~h}$ after treatment. 


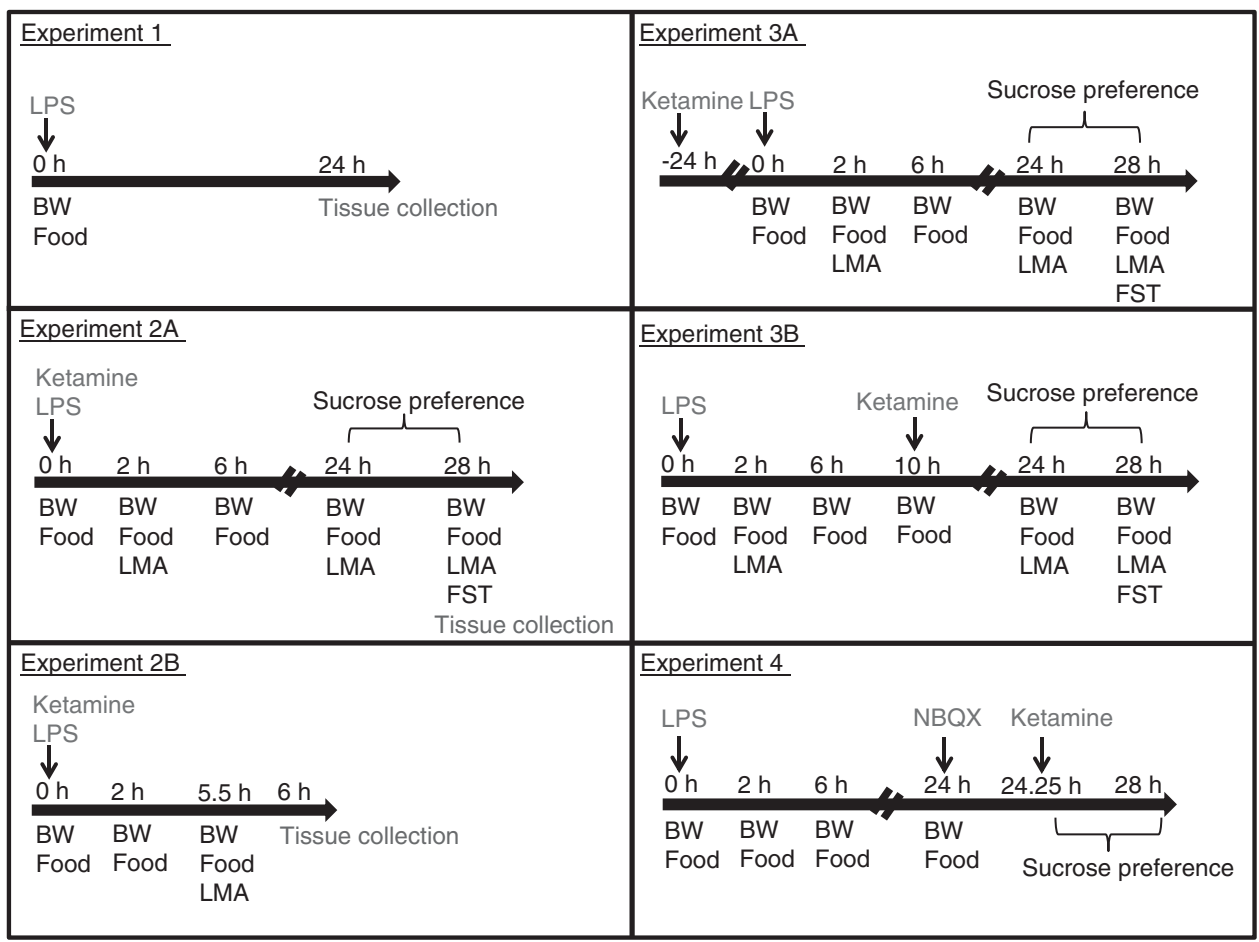

Figure I Experimental timeline and design. BW, body weight; LMA, locomotor activity; FST, forced swim test; LPS, lipopolysaccharide; NBQX, 2,3-dihydroxy-6-nitro-7-sulfamoyl-benzo(f)quinoxaline-2,3-dione.

Forced swim test. At $28 \mathrm{~h}$ after treatment, mice were placed individually into a plastic cylinder (diameter: $23 \mathrm{~cm}$; height: $31 \mathrm{~cm}$ ) containing $15 \mathrm{~cm}$ of water maintained at $24 \pm 1{ }^{\circ} \mathrm{C}$. The same water and bucket were used for a maximum of two mice before being changed. FST lasted for $6 \mathrm{~min}$ and mice were immediately returned to their home cage. The total time spent immobile was scored by a welltrained observer who was blinded to the treatments.

High-performance liquid chromatography (HPLC). IDO activation was indicated by changes in plasma and brain levels of kynurenine and tryptophan, measured by HPLC with electrochemical detection (O'Connor et al, 2009c).

RNA extraction and reverse transcription. Total RNA from liver and whole brain samples were extracted in TRIzol reagent (Life Technologies, Carlsbad, CA). All reverse transcription reactions were performed in a PTC Programmable Thermal Controller (MJ Res, Fitchburg, MA), using an Ambion PureLink RNA Mini reverse transcriptase kit (Life Technologies; catalog number: 12183018A), according to manufacturer's instructions, with random decamer primers for each reaction.

Real-time reverse transcription PCR (RT-PCR). Realtime RT-PCR was carried out on an Applied Biosystems Prism 7900 using TaqMan gene expression assays for interleukin (IL)-6, tryptophan 2,3 dioxygenase (TDO), IDO, BDNF, and glyceraldehyde 3-phosphate dehydrogenase, as previously described (O'Connor et al, 2009a). Given that the BDNF transcript of interest was specific to neurons (exon I-
IX) and is not expressed in the liver, hepatic BDNF was not assessed. See Supplementary Material.

Enzyme-linked immunosorbent assay (ELISA). Plasma IL- $1 \beta$ and IL- 6 were assessed using mouse IL- $1 \beta$ and IL- 6 ELISA sets from BD OptEIA (BD Biosciences, San Jose, CA; catalog number: 559603 and 555240, respectively) according to manufacturer's instructions. Samples were diluted $1: 10(\mathrm{v}: \mathrm{v})$.

Experiment 3: Effect of Ketamine Administered Before or After LPS on LPS-Induced Depressive-Like Behavior

To further confirm that ketamine abrogates depressive-like behavior independently of any effect on LPS-induced inflammation, we ran two timing experiments. Mice were administered ketamine or saline $24 \mathrm{~h}$ before LPS and tested for depressive-like behavior $24 \mathrm{~h}$ after LPS or PBS, when ketamine no longer exerted any NMDA receptor antagonism (experiment 3A). Another group of mice was administered ketamine or saline $10 \mathrm{~h}$ after LPS administration and tested $24 \mathrm{~h}$ after LPS or PBS, when the inflammatory cascade and IDO activation had already developed (experiment $3 \mathrm{~B}$ ).

Experiment 4: Effect of NBQX on Ketamine-Induced Abrogation of LPS-Induced Depressive-Like Behavior

To test the possibility that the antidepressant effects of ketamine are mediated by enhancing AMPA receptor- relative to NMDA receptor-mediated glutamatergic neurotransmission, we administered the AMPA receptor antagonist 2,3dihydroxy-6-nitro-7-sulfamoyl-benzo(f)quinoxaline-2,3-dione (NBQX; $10 \mathrm{mg} / \mathrm{kg}$ diluted in water) subcutaneously $24 \mathrm{~h}$ after 
LPS administration. Ketamine $(6 \mathrm{mg} / \mathrm{kg}$, intraperitoneal) was administered $15 \mathrm{~min}$ thereafter, and mice were tested for sucrose preference during the next $2 \mathrm{~h}$. We decided to focus only on sucrose preference, as this end point is not biased by the alterations in motor activity that may arise because of the combined effects of NBQX and NMDA receptor antagonism (Mead and Stephens, 1998).

\section{Statistical Analysis}

A multivariate analysis of variance (MANOVA) was conducted with LPS treatment as the independent variable and with the kynurenine metabolites as dependent variables for measures of kynurenine metabolites in experiment 1 (LPS $v s$ PBS). Two-way analyses of variances (ANOVAs) (LPS $v s$ PBS $\times$ ketamine $v s$ saline) were performed for all other measures of biochemistry for tissue collected at 6 and $28 \mathrm{~h}$ after treatment. Two-way ANOVAs (LPS $v s$ PBS $\times$ ketamine $v s$ saline) were performed for all measures of depressive-like behavior for the FST and sucrose preference test. Three-way repeated measures ANOVAs (LPS vs PBS $\times$ ketamine vs saline $\times$ time as a repeated factor) were performed for measures of sickness behaviors, body weight loss, and food consumption. Given that LPS has been well documented to induce sickness and depressive-like behavior, and that we expected ketamine to abrogate these effects such that ketamine-treated mice would not exhibit any significant differences to PBS-treated controls, planned comparisons were always performed for the LPS/PBS group against the three other groups. In experiment 4, all groups were compared against the LPS/ketamine/PBS group, as these mice were expected to exhibit significant differences compared with the other three groups. Planned comparisons were determined using $t$-tests with the alpha level of 0.05 adjusted for the number of comparisons to control for familywise error. Only significant comparisons between groups are indicated on figures.

\section{RESULTS}

\section{LPS Activates the Quinolinic Acid Pathway (Experiment 1)}

LPS stimulated the kynurenine pathway in the brain, presumably by activation of kynurenine pathway enzymes, IDO, and kynurenine 3-monooxygenase (Table 1). A one-way MANOVA revealed a significant multivariate main effect for LPS treatment (Wilk's Lamda $=0.033$, $\left.\mathrm{F}_{(13,6)}=13.65, \quad p<0.01\right)$. Univariate analyses indicated that LPS robustly increased kynurenine in the brain $\left(\mathrm{F}_{(1,18)}=15.16, p<0.01\right)$, which was reflected in a significant increase in the brain kynurenine/tryptophan ratio for LPS-treated mice $(m=0.043, \mathrm{SE}=0.003)$ compared with saline-treated controls $\left(m=0.018, \mathrm{SE}=0.001 ; \mathrm{F}_{(1,18)}=8.67\right.$, $p<0.01)$. Levels of brain picolinic acid decreased after LPS treatment $\left(\mathrm{F}_{(1,18)}=20.72, p<0.001\right)$. Increased kynurenine in the brain after LPS treatment was metabolized down the kynurenine 3-monooxygenase branch of the pathway, as shown by LPS-induced elevations in 3-hydroxykynurenine $\left(\mathrm{F}_{(1,18)}=36.62, \quad p<0.0001\right), \quad 3$-hydroxyanthranilic acid $\left(\mathrm{F}_{(1,18)}=36.01, \quad p<0.0001\right)$, and quinolinic acid $\left(\mathrm{F}_{(1,18)}=26.36, p<0.0001\right)$. LPS increased brain tryptophan,
Table I Mean Concentration of Tryptophan and Kynurenine Metabolites ( \pm SEM) in the Brain (pg/mg) for Mice Treated with lipopolysaccharide (LPS) $(n=8)$ or Phosphate-buffered Saline (PBS) $(n=10)$.

\begin{tabular}{lcc}
\hline & \multicolumn{2}{c}{ Brain (pg/mg) } \\
\cline { 2 - 3 } & Saline & LPS \\
\hline Tryptophan & $4316.58(128.14)$ & $5332(161.60)^{* * *}$ \\
Kynurenine & $75.85(15.08)$ & $230.05(20.06)^{* * *}$ \\
3-hydroxykynurenine & $77.50(13.33)$ & $293.60(33.15)^{* * *}$ \\
3-hydroxyanthranilic acid & $0.62(0.12)$ & $1.94(0.18)^{* * * *}$ \\
Quinolinic acid & $3.90(0.36)$ & $12.18(1.57)^{* * *}$ \\
Picolinic acid & $6.59(0.39)$ & $4.25(0.33)^{* *}$ \\
Serotonin & $315.76(15.08)$ & $368.42(17.18)^{*}$ \\
5-hydroxyindoleacetic acid & $266.49(10.16)$ & $418.91(13.61)^{* * *}$ \\
Kynurenic acid & $2.64(0.96)$ & $3.06(0.58)$ \\
Anthranilic acid & $1.03(0.19)$ & $0.73(0.06)$ \\
Xanthurenic acid & $2.05(0.82)$ & $5.35(1.87)$ \\
NAD + & $50347.58(294.26)$ & $51532.79(732.57)$ \\
\hline
\end{tabular}

Abbreviations: KMO, kynurenine 3-monooxygenase; LPS, lipopolysaccharide; $\mathrm{NAD}+$, nicotinamide adenine dinucleotide.

LPS activates the KMO pathway.

Gray shading represents metabolites produced down the $\mathrm{KMO}$ branch. * $p<0.05$, *** $p<0.01$, **** $p<0.0001$.

serotonin, and 5- $\mathrm{HA}\left(\mathrm{F}_{(1,18)}=24.27, p<0.001, \mathrm{~F}_{(1,18)}=5.31\right.$, $p<0.05$, and $\mathrm{F}_{(1,18)}=80.93, p<0.0001$, respectively).

\section{Ketamine Does Not Affect LPS-Induced Sickness (Experiment 2)}

LPS decreased body weight and food consumption across time $\left(\mathrm{F}_{(3,28)}=6.77, p<0.001\right.$ and $\mathrm{F}_{(3,28)}=7.76, p=0.0001$, respectively), and this effect was not altered by ketamine (Supplementary Figure S1A and B).

Mice subjected to tests of depressive-like behavior $24 \mathrm{~h}$ after LPS were first assessed for changes in motor activity 2 and $24 \mathrm{~h}$ after treatment. LPS decreased quadrant entries $\left(\mathrm{F}_{(1,28)}=31.4, p<0.0001\right)$, rears $\left(\mathrm{F}_{(1,28)}=28.5, p<0.0001\right)$, and grooming bouts $\left(\mathrm{F}_{(1,28)}=12.0, p<0.0001\right)$ in a timedependent manner. These effects were not altered by ketamine (Supplementary Figure S2). Planned comparisons revealed that LPS-treated mice reared significantly fewer times at 2 and $24 \mathrm{~h}$ after treatment. LPS-treated mice exhibited significantly fewer grooming bouts and fewer quadrant entries at $2 \mathrm{~h}$, but no differences were observed at the 24-h time point.

Mice allocated for tissue collection $6 \mathrm{~h}$ after treatment were assessed for sickness behavior $5.5 \mathrm{~h}$ after LPS. LPS decreased quadrant entries $\left(\mathrm{F}_{(1,16)}=344.95, p<0.0001\right)$, rears $\left(\mathrm{F}_{(1,16)}=510.527, p<0.0001\right)$, and grooming bouts $\left(\mathrm{F}_{(1,16)}=43.46, p<0.0001\right)$. These effects were not altered by ketamine (Supplementary Figure S2).

\section{Ketamine Abrogates LPS-Induced Depressive-Like Behavior (Experiment 2)}

LPS decreased sucrose preference, and this effect was blocked by ketamine pretreatment. A significant LPS $\times$ ketamine interaction was observed for sucrose consumption between 24 and $28 \mathrm{~h}$ after treatment when ketamine was administered immediately before LPS $\left(\mathrm{F}_{(1,27)}=5.51\right.$, 
$p<0.05$; Figure 2a). Ketamine treatment completely blocked the LPS-induced reduction in sucrose preference, whereby LPS-treated controls exhibited significantly reduced preference for sucrose compared with all other groups (LPS/ PBS group: LPS/ketamine $t_{(13)}=4.38, p<0.05 ; \mathrm{PBS} / \mathrm{PBS}$ $t_{(14)}=3.62, p<0.05$; PBS/ketamine $\left.t_{(14)}=3.25, p<0.05\right)$.

LPS increased the duration of immobility in the FST, an effect that was blocked by ketamine pretreatment. A significant LPS $\times$ ketamine interaction was observed for the time spent immobile $\left(\mathrm{F}_{(1,28)}=4.72, p<0.05\right.$; Figure $\left.2 \mathrm{~b}\right)$. LPS-treated control mice that were not treated with ketamine exhibited significantly greater immobility compared with all other groups (LPS/PBS group: LPS/ketamine $t_{(14)}=3.5, \quad p<0.05 ;$ PBS/PBS $t_{(14)}=2.23, \quad p<0.05$; PBS/ ketamine $\left.t_{(14)}=2.37, p<0.05\right)$.

\section{Ketamine Has No Effect on LPS-Induced Biochemical Alterations (Experiment 2B)}

As expected, LPS increased liver and brain expression of IL- 6 at $6 \mathrm{~h}$ and HO-1 mRNAs at 6 and $28 \mathrm{~h}$ after treatment,
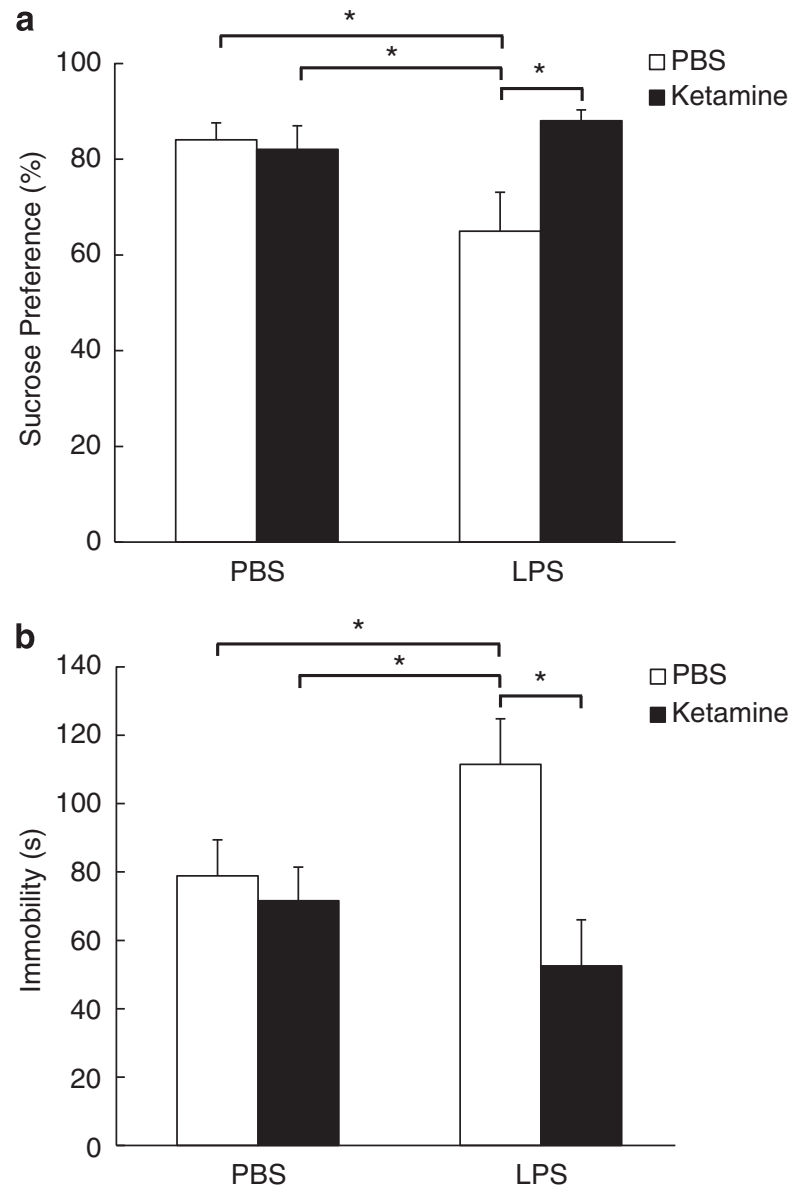

Figure 2 Ketamine abrogates lipopolysaccharide (LPS)-induced depressive-like behavior. (a) Mean sucrose preference (\%) ( \pm SEM) for mice treated with ketamine or phosphate-buffered saline (PBS) immediately before LPS or PBS treatment ( $n \geqslant 7$ per group). (b) Mean time spent immobile in the forced swim test $(s)$ ( \pm SEM) for mice treated with ketamine or PBS immediately before LPS or PBS treatment $(n=8$ per group). Filled bars represent ketamine-treated mice that were treated with either LPS or PBS, and hollow bars represent PBS controls that were treated with either LPS or PBS. $* p<0.05$. and these effects were not affected by ketamine pretreatment (Supplementary Figure S3 and S4). In the same manner, LPS significantly increased plasma levels of IL-1 $\beta$ at $6 \mathrm{~h}$ and of IL- 6 at 6 and $28 \mathrm{~h}$ after treatment, and these effects that were not altered by ketamine (Supplementary Figure S5). Essentially the same pattern was observed for IDO mRNA in the brain and liver (Supplementary Figure S6). Expression of the other tryptophan-degrading enzyme, TDO, in the brain and liver also increased significantly by LPS at $6 \mathrm{~h}$ after treatment (Supplementary Figure S7). TDO mRNA was back to baseline levels in the liver and was decreased in the brain at $28 \mathrm{~h}$ after treatment.

LPS increased plasma kynurenine/tryptophan ratios at 6 and $28 \mathrm{~h}$ after treatment. Brain kynurenine/tryptophan ratios were elevated in LPS-treated mice $28 \mathrm{~h}$ after treatment. Ketamine had no effect alone or in interaction with LPS (Supplementary Figure S8).

Brain BDNF mRNA was decreased in LPS-treated mice at $6 \mathrm{~h}$ but not $28 \mathrm{~h}$ after treatment, and this effect was not modified by ketamine (Supplementary Figure S9). Detailed statistics for all these biochemical alterations from LPS are provided in Supplementary Material.

Ketamine Administered $24 \mathrm{~h}$ before LPS Did Not Affect LPS-Induced Depressive-Like Behavior in Contrast to Ketamine Administered $10 \mathrm{~h}$ After LPS (Experiment 3)

Ketamine administered $24 \mathrm{~h}$ before LPS did not interact with LPS-induced reductions in sucrose preference and increased immobility in the FST $\left(\mathrm{F}_{(1,23)}=5.79, p<0.05\right.$ and $\mathrm{F}_{(1,22)}=4.30, p=0.05$, respectively) (Figure $3 \mathrm{a}$ and $\mathrm{b}$ ). Ketamine administered $10 \mathrm{~h}$ after LPS abrogated LPSinduced reductions in sucrose preference $\left(\mathrm{F}_{(1,23)}=9.38\right.$, $p<0.01$; LPS/PBS $v s$ LPS/ketamine mice $t_{(11)}=2.98$, $p<0.05 ;$ LPS/PBS vs PBS/PBS mice $t_{(12)}=2.93, p<0.05$; Figure $3 \mathrm{c}$ ). In both cases, ketamine had no effect on LPSinduced sickness (Supplementary Figure S10-S12).

\section{NBQX Abrogates the Alleviating Effect of Ketamine on LPS-Induced Depressive-Like Behavior (Experiment 4)}

To assess the ability of ketamine to elevate AMPA receptormediated glutamatergic transmission relative to NMDA receptor-mediated glutamatergic transmission, the AMPA receptor antagonist NBQX was administered $24 \mathrm{~h}$ after LPS and $15 \mathrm{~min}$ before ketamine. Sucrose preference was assessed during the next $2 \mathrm{~h}$.

A significant NBQX $\times$ ketamine interaction was observed for LPS-induced reductions in sucrose preference $\left(F_{(1,23)}=8.97, p<0.01\right)$. Ketamine abrogated LPS-induced decreased sucrose preference (LPS/PBS/ketamine $v s$ LPS/PBS/CTRL $\left.t_{(12)}=2.95, p<0.05\right)$, which was restored in the presence of the AMPA antagonist NBQX (LPS/NBQX/ketamine $v s$ LPS/NBQX/CTRL $t_{(11)}=2.8$, $p<0.05$; Figure 4). NBQX and ketamine had no effect on LPS-induced sickness measures (Supplementary Figure S10 and S11).

\section{DISCUSSION}

The present findings show that LPS increases the levels of the NMDA receptor agonist quinolinic acid in the brain. 

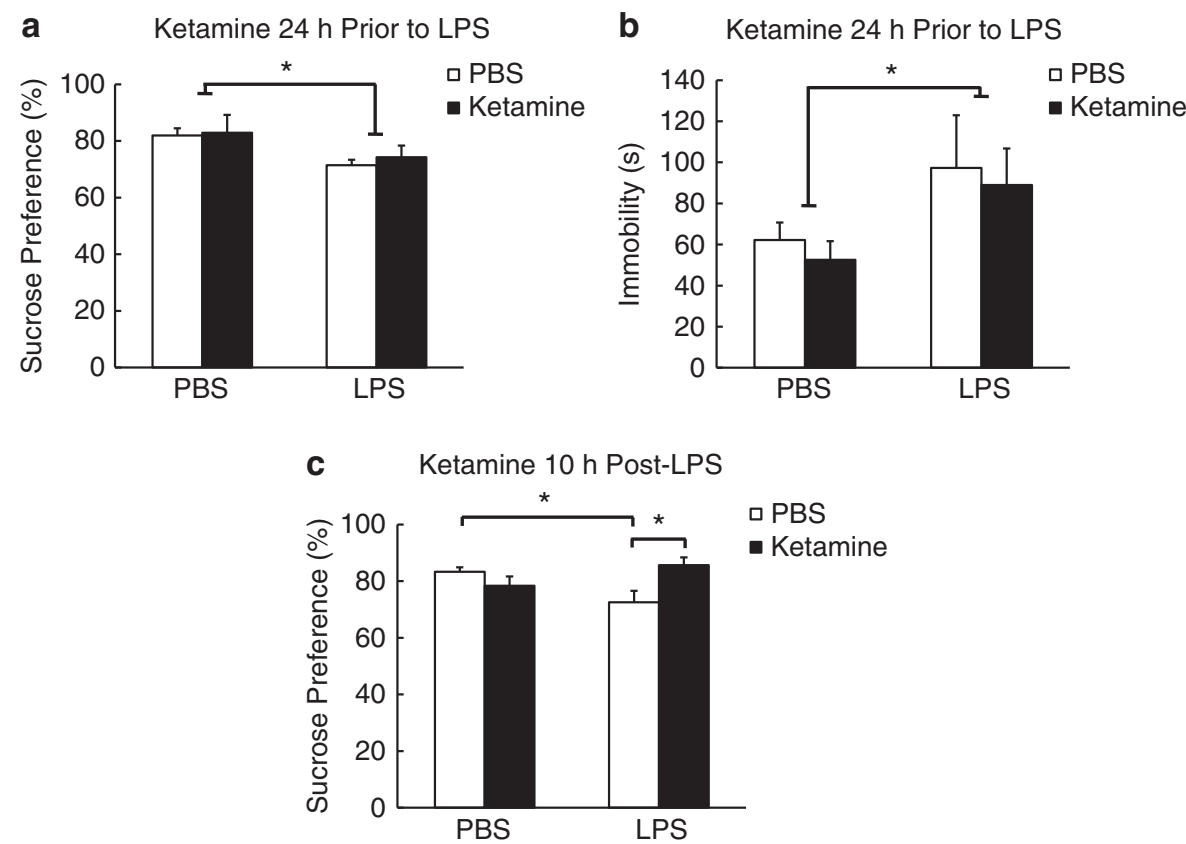

Figure 3 Ketamine treatment $10 \mathrm{~h}$ after administration of lipopolysaccharide (LPS) but not $24 \mathrm{~h}$ before abrogates LPS-induced depressive-like behavior. ( $a$ and b) Mean sucrose preference (\%) ( \pm SEM) and time spent immobile in the forced swim test (s) ( \pm SEM) for mice treated with ketamine or phosphatebuffered saline (PBS) $24 \mathrm{~h}$ before LPS or PBS treatment ( $n \geqslant 6$ for all groups). (c) Mean sucrose preference (\%) ( \pm SEM) for mice treated with ketamine or PBS $10 \mathrm{~h}$ after LPS or PBS treatment ( $n \geqslant 6$ for all groups). Filled bars represent ketamine-treated mice that were treated with either LPS or PBS, and hollow bars represent PBS controls that were treated with either LPS or PBS. $* p<0.05$.

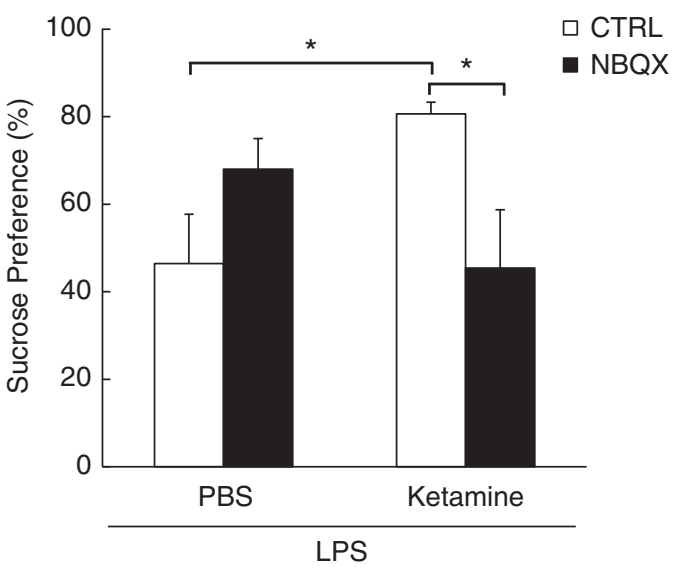

Figure 4 NBQX (2,3-dihydroxy-6-nitro-7-sulfamoyl-benzo(f)quinoxaline-2,3-dione) restores reduced sucrose preference in lipopolysaccharide (LPS)-treated mice administered ketamine. Mean sucrose preference (\%) $( \pm$ SEM) for LPS-treated mice treated with ketamine or phosphatebuffered saline (PBS) I 5 min after NBQX or control treatment $(n \geqslant 6$ per group). Filled bars represent NBQX-treated mice that were treated with either ketamine or PBS, and hollow bars represent controls that were treated with either ketamine or PBS. ${ }^{*} p<0.05$.

The NMDA receptor antagonist ketamine abrogates LPSinduced depressive-like behavior when administered just before LPS or well after LPS, once inflammation and IDO activation have developed. This effect is not due to any interference of ketamine with LPS-induced inflammation and BDNF decrease, and it is observed at a dose of ketamine that has no antidepressant-like effect of its own. The blockade of LPS-induced reduction in sucrose preference is antagonized by pretreatment with the AMPA receptor antagonist NBQX.

The activation of IDO by LPS induces the formation of kynurenine, both at the periphery and in the brain (O'Connor et al, 2009c). In the brain, locally produced kynurenine and kynurenine transported from the periphery are metabolized along two pathways; one pathway involving kynurenine aminotransferases, which results in the formation of the NMDA receptor antagonist kynurenic acid, and another pathway involving kynurenine 3 -monooxygenase that leads to the formation of 3-hydroxykynurenine and quinolinic acid. In response to LPS, the formation of 3-hydroxykynurenine and quinolinic acid predominates over that of kynurenine, in a similar manner to what was already described in response to pokeweed mitogen (Saito et al, 1992). This is probably because of the activation of microglial cells that contain kynurenine 3-monooxygenase, as suggested by the demonstration that pretreatment with minocycline, a potent downregulator of microglial activation, abrogates the development of LPS-induced depressive-like behavior (Connor et al, 2008). The observed increase in brain levels of tryptophan and serotonin metabolism in response to LPS have already been described (Dunn and Welch, 1991). This increase in serotonin metabolism has been interpreted as a stress response (Dunn et al, 1999) and is not specific to the LPS-induced depressive-like behavior observed in this study.

In view of the increased brain levels of 3-hydroxykynurenine and quinolinic acid in response to LPS, it could be speculated that LPS-induced depressive-like behavior is mediated by oxidative stress generated by the potent radical donor 3-hydroxykynurenine or by activation of NMDA 
receptor glutamatergic neurotransmission by quinolinic acid. We used the NMDA receptor antagonist ketamine to determine whether activation of NMDA receptor glutamatergic neurotransmission in response to LPS is sufficient to induce depressive-like behavior. We observed that ketamine administration is able to fully abrogate LPS-induced depressive-like behavior.

Along with its NMDA receptor antagonist properties, ketamine has a number of off-target side effects, including inhibition of inflammation by upregulation of HO-1 (Hoetzel and Schmidt, 2010). The dose of ketamine we administered did not interfere with the induction of HO-1 at the periphery and in the brain in response to LPS. In accordance with this lack of effect of ketamine, we did not observe any effect of this compound on the inflammatory response to LPS at the periphery and in the brain. In the absence of any regional analysis of cytokine expression in the brain, we are not able to discard the possibility that ketamine alters the expression of cytokines in specific brain areas. However, such a possibility is unlikely as a region-specific anti-inflammatory activity of ketamine should have been accompanied by decreased sickness behaviors, as indicated by alleviation of reduced food intake, body weight loss, and locomotor activity, which was clearly not the case. The lack of effect of ketamine on sickness behavior indicates a predominant effect of this compound downstream of the inflammatory cascade triggered by LPS.

Activation of IDO and other enzymes involved in the formation of kynurenine metabolites is another possible target for ketamine antidepressant-like activity in LPStreated mice. Immune activation activates IDO and TDO (O'Connor et al, 2009a, b, c; Park et al, 2011), with the increase in the activity of this last enzyme probably being mediated indirectly by increased glucocorticoid receptor activation (Bhagwagar et al, 2003; Pariante and Lightman, 2008). We did not find any evidence for an interference of ketamine with the formation of kynurenine in the brain, as illuminated by the lack of variation in the kynurenine/ tryptophan ratio in ketamine-treated mice. We did not investigate the effects of ketamine on the production of kynurenine metabolites downstream of IDO. In particular, we did not assess whether ketamine switches the metabolism of kynurenine from the kynurenine 3-monooxygenase pathway to the kynurenine aminotransferase pathway to result in an increase in brain levels of kynurenic acid. However, such an effect is unlikely as the results of the NBQX experiment discussed below do not support the possibility of ketamine-induced alterations in the balance between kynurenic acid and quinolinic acid, and ketamine is able to antagonize LPS-induced depressive-like behavior even when administered well after IDO and other enzymes of the tryptophan degrading pathway have been activated in response to LPS, $24 \mathrm{~h}$ after LPS in the NBQX experiment.

The enhanced production of brain BDNF downstream of NMDA receptor blockade by ketamine could have been responsible for the antidepressant-like activity of ketamine on LPS-induced depressive-like behavior in the context of a LPS-induced decrease of BDNF. Ketamine has been shown to increase BDNF in the hippocampus and prefrontal cortex (Garcia et al, 2008; Ibla et al, 2009; Autry et al, 2011).
NMDA blockade by ketamine deactivates eukaryotic elongation factor 2 (eEF2) kinase, resulting in reduced eEF2 phosphorylation and desuppression of rapid dendritic protein translation, including BDNF (Monteggia et al, 2012). We did not investigate this pathway directly but restricted ourselves to the consideration of the possible alleviating effects of ketamine on LPS-induced decreases in brain BDNF. Ketamine did not alter LPS-induced decreases of BDNF at $6 \mathrm{~h}$ after treatment, probably because the acute stimulating effect of ketamine on BDNF had already ended (Autry et al, 2011).

To confirm that the antidepressant-like activity of ketamine is specific to LPS and occurs via blockade of NMDA glutamatergic receptor signaling, independently of LPS-induced inflammation and production of kynurenine metabolites, we undertook two additional experiments. First, we showed that ketamine's antidepressant-like activity occurred apparently well after the inflammatory and IDO response to LPS had fully developed. We verified that ketamine injected $24 \mathrm{~h}$ before LPS administration ( $48 \mathrm{~h}$ before depression testing) fails to abrogate the onset of LPSinduced depressive-like behaviors. Then, we injected ketamine $10 \mathrm{~h}$ after LPS, when the inflammatory and kynurenine pathways had already been activated. In this condition, ketamine was able to abrogate LPS-induced depressive-like behaviors. The same effect of ketamine was obtained when the injection of ketamine was delayed up to $24 \mathrm{~h}$ after LPS.

Second, we directly tested the hypothesis that ketamine abrogates LPS-induced depressive-like behavior by antagonizing NMDA receptor activation. In addition to blocking the NMDA receptor, ketamine also promotes AMPAmediated glutamatergic neurotransmission, which can reduce depressive-like symptoms (Maeng and Zarate, 2007; Maeng et al, 2008). There is already evidence that the antidepressant-like activity of ketamine is blocked by the AMPA antagonist NBQX (Maeng and Zarate, 2007; Maeng et al, 2008; Autry et al, 2011). We therefore administered NBQX to LPS-treated mice, 15 min before ketamine injection. As predicted, this treatment resulted in the restoration of the depressive-like phenotype in the sucrose preference test in ketamine-treated mice. In summary, the findings of the present experiments indicate that LPS induces depressive-like behavior by activating NMDA receptors, and this effect is probably mediated by the increased brain production of the NMDA receptor antagonist quinolinic acid. These results further add to our understanding of the mechanisms of inflammation-induced depression.

\section{ACKNOWLEDGEMENTS}

We thank Marcus A Lawson, Robert H McCusker, and Scott E Nixon for their assistance and input. This work was supported by National Institutes of Health (NIH) Grants to RD (R01 MH079829) and KWK (R01 AG029573), NIH Grant RO1 NS073939, and in part by MD Anderson Cancer Center Support Grant CA016672. The content is solely the responsibility of the authors and does not necessarily represent the official views of the National Institutes of Health. 


\section{DISCLOSURE}

Drs Dantzer and Kelley are funded by the National Institutes of Health (NIH). Dr Dantzer has received recent honoraria from Lundbeck Laboratories and Janssen. He was also a consultant for Lundbeck Laboratories. Dr Budac, Dr Lee, and Ms Bisulco are employed by Lundbeck Laboratories. The remaining authors report no conflicts of interest.

\section{REFERENCES}

Autry AE, Adachi M, Nosyreva E, Na ES, Los MF, Cheng PF et al (2011). NMDA receptor blockage at rest triggers rapid behavioural antidepressant responses. Nature 475: 91-95.

Bhagwagar Z, Hafizi S, Cowen PJ (2003). Increase in concentration of waking salivary cortisol in recovered patients with depression. Am J Psychiatry 160: 1890-1891.

Capuron L, Gumnick JF, Musselman DL, Lawson DH, Reemsnyder A, Nemeroff CB et al (2002). Neurobehavioral effects of interferon- $\alpha$ in cancer patients: phenomenology and paroxetine responsiveness of symptom dimensions. Neuropsychopharmacol 26: $643-652$.

Connor TJ, Starr N, O'Sullivan JB, Harkin A (2008). Induction of indolamine 2,3-dioxygenase and kynurenine 3-monooxygenase in rat brain following a systemic inflammatory challenge: a role for IFN- $\gamma$ ? Neurosci Lett 441: 29-34.

Dantzer R, O'Connor JC, Freund GG, Johnson RW, Kelley KW (2008). From inflammation to sickness and depression: when the immune system subjugates the brain. Nat Rev Neurosci 9: 46-56.

Dunn AJ, Wang J, Ando T (1999). Effects of cytokines on cerebral neurotransmission. Comparison with the effects of stress. $A d v$ Exp Med Biol 461: 117-127.

Dunn AJ, Welch J (1991). Stress- and endotoxin-induced increases in brain tryptophan and serotonin metabolism depend on sympathetic nervous system activity. J Neurochem 57: 1616-1622.

Frenois F, Moreau M, O'Connor J, Lawson M, Micon C, Lestage J et al (2007). Lipopolysaccharide induces delayed FosB/DeltaFosB immunostaining within the mouse extended amygdala, hippocampus and hypothalamus, that parallel the expression of depressive-like behavior. Psychoneuroendocrinol 32: 516-531.

Garcia LS, Comim CM, Valvassori SS, Réus GZ, Barbosa LM, Andreazza AC et al (2008). Acute administration of ketamine induces antidepressant-like effects in the forced swimming test and increases BDNF levels in the rat hippocampus. Prog Neuropsychopharmacol Biol Psychiatry 32: 140-144.

Guan Z, Fang J (2006). Peripheral immune activation by lipopolysaccharide decreases neurotrophins in the cortex and hippocampus in rats. Brain Behav Immun 20: 64-71.

Guillemin GJ, Kerr SJ, Smythe GA, Smith DG, Kapoor V, Armati PJ et al (2001). Kynurenine pathway metabolism in human astrocytes: a paradox for neuronal protection. J Neurochemistry 78: $842-853$.

Heyes MP, Achim CL, Wiley CA, Major EO, Saito K, Markey SP (1996). Human microglia convert L-tryptophan into the neurotoxin quinolinic acid. Biochem J 320: 595-597.

Hoetzel A, Schmidt R (2010). Regulatory role of anesthetics on heme oxygenase-1. Curr Drug Targets 11: 1495-1503.

Ibla JC, Hayashi H, Bajic D, Soriano SG (2009). Prolonged exposure to ketamine increases brain derived neurotrophic factor levels in developing rat brains. Curr Drug Saf 4: 11-16.

Maeng S, Zarate CA Jr (2007). The role of glutamate in mood disorders: results from the ketamine in major depression study and the presumed cellular mechanism underlying its antidepressant effects. Curr Psychiatry Rep 9: 467-474.

Maeng S, Zarate CA Jr, Du J, Schloesser RJ, McCammon J, Chen G et al (2008). Cellular mechanisms underlying the antidepressant effects of ketamine: role of $\alpha$-amino-3-hydroxy-5-methylisoxazole-4-propionic acid receptors. Biol Psychiatry 63: 349-352.

Mead AN, Stephens DN (1998). AMPA-receptors are involved in the expression of amphetamine-induced behavioural sensitisation, but not in the expression of amphetamine-induced conditioned activity in mice. Neuropharmacol 37: 1131-1138.

Monteggia LM, Gideons E, Kavalali ET (2012). The role of eukaryotic elongation factor 2 kinase in rapid antidepressant action of ketamine. Biol Psychiatry S0006-3223: 00778-0.

Moreau M, André C, O'Connor JC, Dumich SA, Woods JA, Kelley $\mathrm{KW}$ et al (2008). Inoculation of Bacillus Calmette-Guerin to mice induces an acute episode of sickness behavior followed by chronic depressive-like behavior. Brain Behav Immun 22: 1087-1095.

Mormède C, Palin K, Kelley KW, Castanon N, Dantzer R (2004). Conditioned taste aversion with lipopolysaccharide and peptidoglycan does not activate cytokine gene expression in the spleen and hypothalamus of mice. Brain Behav Immun 18: 186-200.

O'Connor JC, André C, Wang Y, Lawson MA, Szegedi SS, Lestage J et al (2009a). Interferon- $\gamma$ and tumor necrosis factor- $\alpha$ mediate the upregulation of indoleamine 2,3-dioxygenase and the induction of depressive-like behavior in mice in response to bacillus Calmette-Guerin. J Neurosci 29: 4200-4209.

O'Connor JC, Lawson MA, André C, Briley EM, Szegedi SS, Lestage J et al (2009b). Induction of IDO by Bacille Calmette-Guérin is responsible for development of murine depressive-like behavior. J Immunol 182: 3202-3212.

O'Connor JC, Lawson MA, André C, Moreau M, Lestage J, Castanon $\mathrm{N}$ et al (2009c). Lipopolysaccharide-induced depressive-like behavior is mediated by indoleamine 2,3-dioxygenase activation in mice. Mol Psychiatry 14: 511-522.

Pariante CM, Lightman SL (2008). The HPA axis in major depression: classical theories and new developments. Trends Neurosci 31: 464-468.

Park SE, Lawson M, Dantzer R, Kelley KW, McCusker RH (2011). Insulin-like growth factor-I peptides act centrally to decrease depression-like behavior of mice treated intraperitoneally with lipopolysaccharide. J Neuroinflamm 8: 179.

Reichenberg A, Yirmiya R, Schuld A, Kraus T, Haack M, Morag A et al (2001). Cytokine-associated emotional and cognitive disturbances in humans. Arch Gen Psychiatry 58: 445-452.

Saito K, Markey SP, Heyes MP (1992). Effects of immune activation on quinolinic acid and neuroactive kynurenines in the mouse. Neurosci 51: 25-39.

Serrats J, Schiltz JC, García-Bueno B, van Rooijen N, Reyes TM, van Dam AM et al (2010). Dual roles for perivascular macrophages in immune-to-brain signaling. Neuron 65: 94-106. van Dam AM, Brouns M, Louisse S, Berkenbosch F (1992). Appearance of interleukin-1 in macrophages and in ramified microglia in the brain of endotoxin-treated rats: a pathway for the induction of non-specific symptoms of sickness? Brain Res 588: 291-296.

Wright CE, Strike PC, Brydon L, Steptoe A (2005). Acute inflammation and negative mood: mediation by cytokine activation. Brain Behav Immun 19: 345-350.

Wu SY, Wang TF, Yu L, Jen CJ, Chuang JI, Wu FS et al (2011). Running exercise protects the substantia nigra dopaminergic neurons against inflammation-induced degeneration via the activation of BDNF signaling pathway. Brain Behav Immun 25: 135-146.

Supplementary Information accompanies the paper on the Neuropsychopharmacology website (http://www.nature.com/npp) 